\title{
sciendo
}

\section{Effect of Different Feedback Modalities on Swimming Pace: Which Feedback Modality is Most Effective?}

\author{
by \\ Cesare Altavilla ${ }^{1}$, Roberto Cejuela ${ }^{2}$ Pablo Caballero-Pérez ${ }^{3}$
}

\begin{abstract}
To compare the effect of three different feedback modalities on swimming pace, sixteen male swimmers and triathletes participated in this study. Each participant swam $3 \times 400 \mathrm{~m}$, one for each feedback modality, swimming front crawl at $80 \%$ of their individual swimming critical speed. Three feedback modalities were examined: self-pacing, real time visual feedback and real time voice feedback. The swimmers adopted a fast start in all feedback modalities. In the real time voice feedback modality, the data recorded during the second lap $(200 \mathrm{~m})$ showed a significant improvement of their swimming pace approaching the swimming pace intended (-1.47s, $p<.01$, medium effect size 0.79). A significant improvement toward the swimming pace intended was also noticed at the third split time $(300 \mathrm{~m})(0.05 \mathrm{~s}, \mathrm{p}<.01$, large effect size 0.81) and at the fourth split time $(400 \mathrm{~m})(0.46 \mathrm{~s}, p<.01$, medium effect size 0.76). In self-pacing, the swimmers were not able to swim in line with the swimming pace intended. In real time visual feedback modality, the swimmers did not show a significant improvement approaching the swimming pace intended. The results revealed that communication with the swimmers using the real time voice feedback induced a significant improvement in their swimming pace and could help the athletes to swim with accurate and consistent pace.
\end{abstract}

Key words: training, performance, auditory pathways, sensory, split time.

\section{Introduction}

Nowadays, science and new technology strongly influence many sports. Over the past 30 years, a substantial amount of research has contributed to improving swimming science and its application in training is becoming indispensable. Despite aquatic conditions, the technological progress has allowed the development of some informative instruments that assist swimmers during and after performing a task (Perez et al., 2009). Most of these instruments do not allow real time communication between the coach and the swimmer and some of them need to have the registered data processed after the training session. Real time feedback is important in swimming because the "feeling" about your own performance is usually inaccurate. It is a known fact that the swimmer's personal feeling of her/his own swimming pace does not always coincide with the external evaluation. Therefore, in swimming, new instruments should allow real time communication between the coach and the swimmer, and at the same time they must be wearable and should not interfere with swimmer's performance (Bächlin and Tröster, 2012; Turner et al., 2008). It is speculated that the regulation of exercise pace is dictated by a combination of the ability to resist fatigue, feedback integration, anticipatory forecasting and indeed previous experience (Tucker and Noakes, 2009). However, the responsible mechanisms are currently unclear and it remains a controversial

\footnotetext{
1 - Foods analysis and nutrition group, University of Alicante, Alicante, Spain.

2 - Department-section of Physical Education and Sport, University of Alicante, Spain.

3 - Department of Community Nursing, Preventive Medicine, Public Health and History of Science, University of Alicante, Alicante, Spain.
} 
issue what constitutes well-executed work pace. Observation of changes in the velocity pattern during competitions has developed an increasing interest in pacing strategy during athletic competitions (de Koning et al., 2011; Mytton et al., 2015). Pacing strategy can be defined as the goal directed distribution and management of effort across the duration of an exercise bout (Edwards and Polman, 2012). The effect of pacing on performance has been studied in many cyclic sports, including swimming (Thompson et al., 2004), cycling (Abbiss et al., 2013), running (Bath et al., 2012) and triathlon (Le Meur et al., 2009; Wu et al., 2015).

Swimming pace can be defined as the speed at which a swimmer completes a distance and indeed is an important variable to be monitored to obtain a better performance (Scruton et al., 2015; Thompson, 2014). The sport of swimming is biomechanically inefficient with high drag force, therefore, appropriate swimming pace is crucial to avoid any undesirable onset of sudden fatigue, which will determine loss of stroke power, coordination and speed (Mauger et al., 2012; Thompson, 2014). It has been reported that the ability to repeat constant swimming pace in different conditions is a quality of competitive and elite swimmers (Skorski et al., 2013, 2014). There is no widespread acceptance of any pacing method for swimming. Traditionally, coaches manage the swimming pace by giving visible coded signals to swimmers with their hands, while standing on the poolside. Some studies have reported that the modalities of feedback given to the swimmer could affect swimming pace (Perez et al., 2009; Turner et al., 2008). Few studies have attempted to use audio pacing signals to communicate swimming pace to the swimmer (Thompson et al., 2002, 2004). We did not find any study where some instruction was given by an instrument that allowed the coach to speak in real time to the swimmer. Moreover, we did not find any study where real time voice feedback was compared to other feedback modalities on swimming pace.

The aim of this study was to compare swimming pace under three different feedback conditions: no feedback or self-pacing, with real time visual feedback or with real time voice feedback. We hypothesized that real time voice feedback would be an effective way to communicate with the swimmer.

\section{Methods}

\section{Participants}

Sixteen males participated voluntarily in the study. We selected different levels of experienced swimmers and triathletes with a minimum of two years of sport experience, including 5 swimmers and triathletes that competed in national championships (age $19.70 \pm$ 2.17 years old; body height $176.50 \pm 5.99 \mathrm{~cm}$; body mass $70.18 \pm 4.82 \mathrm{~kg}$ ). We chose participants with heterogeneous experience and did not select only swimmers or triathletes of elite national level, because well-executed swimming pace has a huge impact at all levels of swimming and triathlon training and competitions. In this study swimmers and triathletes are both called swimmers.

\section{Measures}

Each swimmer performed $3 \times 400 \mathrm{~m}$, one for each feedback modality, using a front crawl style in a $25 \mathrm{~m}$ swimming pool at the swimming pace intended. The swimming pace intended was $80 \%$ of their individual swimming critical speed (CSS), which is within an aerobic range (Touretski, 1993). The start of every trial was given with wall push off. Prior to the study the CSS of each swimmer was determined according to the protocol described by Ginn (1993). CSS can be defined as the highest sustainable work rate which enables lactate to remain at a steady-state (Ginn, 1993). CSS is an inexpensive and noninvasive method commonly used to easily evaluate and adjust training pace (Dekerle et al., 2002). The study participants swam with three feedback conditions: self-pacing, real time visual feedback and real time voice feedback with 1 week intervals between the three trials. The swimmer was informed about the swimming pace intended before starting swimming, and further feedback was given every $100 \mathrm{~m}$. Before each trial athletes swam between 1000 and $1500 \mathrm{~m}$ for a warm-up consisting of free continuous mixed swim at moderate intensity. The swimmers repeated the same in-water warm-up before each trial. Between the warm-up and the trial, the swimmer was given at least 5-10 min of complete rest.

Swimmers were requested to refrain from any high intensity exercise at least 24 hours before 
the trials. They were also asked not to consume food for the 2 hours before the trials and abstain from alcohol, caffeine or any other energy drink on the day of each trial. All the swimmers had reported no hearing or visual issues in the 12 months prior to the beginning of the study.

\section{Procedures}

All the modalities were provided to the swimmers over the three trials, but only one of the three modalities was given on a trial. The data was recorded at the beginning of training after the warm-up. There was no familiarization trial because self-pacing and real time visual feedback modalities are used daily during training. The swimmers were informed about their split time during the trials. During every trial, except selfpacing, swimmers were informed about their swimming pace every $100 \mathrm{~m}$, specifically, the difference in seconds with respect to the swimming pace intended was communicated to the swimmers. In order to evaluate the effects of the three feedback modalities on swimming pace, in every trial a split time every $100 \mathrm{~m}$ and a total time were recorded. One swim coach experienced in timekeeping was the time keeper for each swimmer. Timekeeping was recorded by a stopwatch 3X - 100 (Finis, Livermore, CA, USA). The real time feedback was provided every $100 \mathrm{~m}$, between $5-10 \mathrm{~m}$ after the swimmer made the turn. The real time visual feedback consisted of a code of signals-gestures with one's hands, which were explained to the swimmer before the trial session and normally used by the coaches on the poolside. In this study the real time voice feedback consisted of a short voice message, which was given to the swimmer via the Ucoach device (S.H.O.T. Swimmers Hit On Technology, Rome, Italy). This device is a swim training tool designed to talk to the swimmer during a training session providing real time voice feedback. The system consists of a poolside transmitter and a waterproof receiver, the first one allows the coach to communicate in real time, by a small microphone to minimize ambient sound. The waterproof receiver easily fits under any kind of a swimming cap and the voice of the coach is perceived by the swimmer from the headset via moving bone conduction technology. All swimmers were informed verbally and in writing about the nature of the study, including all potential risks. The swimmers signed a written informed consent form prior to the beginning of the study and the ethics committee of the University of Alicante granted ethical approval, according to the Declaration of Helsinki.

\section{Statistical Analysis}

Every $100 \mathrm{~m}$ difference in seconds between the pre-set speed and the one obtained under the three modalities of feedback was recorded. A difference of zero seconds indicated perfect adaptation to the speed desired, a negative value meant faster pace and a positive value meant slower pace. Standard error and confidence intervals were calculated. In all three tests, the first $100 \mathrm{~m}$ were swum without providing information about athletes' pace. In order to distinguish between the feedback modalities during the 200, 300 and 400 subsequent $\mathrm{m}$, the differences with the swimming pace intended were compared at all times. In addition, each feedback modality was analyzed to determine which feedback was most efficient in helping the swimmers in matching the pace. Standard deviation (SD) of the difference regarding the swimming pace intended could be considered as the measure of accuracy of each feedback modality. Therefore, the differences among the SDs at 200, 300 and $400 \mathrm{~m}$ between the three feedback modalities were calculated. We evaluated the effect size as a fraction of the variability between participants. We classified effect size as trivial (0-0.19) small (0.2-0.49), medium (0.5-0.79) and large (0.8 and greater) (Cohen, 1992). Effect size was calculated to determine the smallest worthwhile differences in the trials. To compare and analyze the differences between each modality and between the various distances the paired two-sample t-test was carried out. Not being able to guarantee normal statistical distribution, its equivalent, non-parametric Wilcoxon test was performed. To analyze the differences between the SDs we used the F-test of the equality of two variances, taking into account the robustness of the F-test with respect to equality of sample size. The SPSS 15.0 was used to carry out this analysis.

\section{Results}

The mean pace difference of the three different feedback modalities, comparing the swimming pace intended, is shown in Table 1. In all the three modalities, the swimmers had no information within the first $100 \mathrm{~m}$ of the $400 \mathrm{~m}$ 
effort and there was no significant difference in swimming pace of the first split time, between the three modalities. With regard to the pacing pattern, the swimmers adopted a fast start in all the modalities. In the self-pacing modality and in the real time voice feedback modality, $68.75 \%$ of the swimmers adopted a fast start, meanwhile in the real time visual feedback modality it was $87.5 \%$. The results revealed that at $100 \mathrm{~m}$ the swimmers swam between 2.87 (real time voice feedback modality) and $4.73 \mathrm{~s}$ (real time visual feedback modality) faster than the swimming pace intended (Table 1).

The swimmers in real time voice feedback modality were able to reach swimming pace close to the intended one. In this feedback modality, throughout the $400 \mathrm{~m}$ swim, the participants gradually swam closer to the swimming pace intended, whereas in self-pacing and in real time visual feedback such improvement was not revealed (Figure 1). In the real time feedback modalities as soon as the first split time $(100 \mathrm{~m})$ was recorded, it was quickly communicated to the swimmer. In the real time voice feedback the data recorded during the second lap $(200 \mathrm{~m})$ showed a significant correction of athletes' swimming pace approaching the swimming pace intended $(p<$ .01). A significant correction toward the swimming pace intended was also noticed at the third $(300 \mathrm{~m})(p<.01)$ and the fourth split time $(400 \mathrm{~m})(p<.05)$.

In self-pacing, the participants were not able to swim in line with the swimming pace intended, they adopted a fast start and during the $400 \mathrm{~m}$ they did not correct their swimming pace (Figure 1, Table 1). In real time visual feedback modality, after the hand signals-gestures at $100 \mathrm{~m}$, the swimmers did not show a significant correction approaching the swimming pace intended at the second split time $(200 \mathrm{~m})$, neither in the next split times at $300 \mathrm{~m}$ and $400 \mathrm{~m}$ (Figure 1, Table 1). Surprisingly, the real time visual feedback was less effective than the rest of modalities in terms of times closest to swimming pace intended (Figure 1), but it was difficult to ensure that swimmers were able to see the visual feedback given during the trial. We also compared the mean difference with swimming pace intended between feedback modalities every 100 $\mathrm{m}$, and at $300 \mathrm{~m}$ and $400 \mathrm{~m}$ split times we recorded a significant difference between real time voice feedback and real time visual feedback $(p=.05)$ (Figure 1, Table 1).

In real time voice feedback, medium and large effect size was recorded according to Cohen's classification (Table 1). Meanwhile trivial effect size was recorded in self-pacing and real time visual feedback (Table 1). Table 2 shows the estimated SD values relative to the pace of each feedback modality. Figure 2 graphically shows an increase in the variability of the SD in self-pacing and real time visual feedback. Self-pacing and real time visual feedback show higher values at $200 \mathrm{~m}$ with meaningful differences between $100 \mathrm{~m}$ and $200 \mathrm{~m}(p<.05)$.

Instead, with real time voice feedback, SD values did not show any substantial changes during the whole trial (Figure 2), even if with this feedback modality the initial value at $100 \mathrm{~m}$ was highest when compared with the other two modalities. Furthermore, the differences between $\mathrm{SD}$ values of the real time voice feedback and of self-pacing at $300 \mathrm{~m}(p<.05)$ and $400 \mathrm{~m}(p<.01)$ were statistically meaningful.

All the swimmers reported an additional motivational boost with the real time voice feedback modality, when they heard the voice of the speaker via the Ucoach device. We do not have any objective measurements about the drag forces produced by the Ucoach receiver, but all participants declared that the receiver was quite comfortable to wear. The subjective feedback, given by the swimmers, suggests that the Ucoach receiver did not compromise swimming performance with any additional water resistance or by disturbing fluid dynamics.

\section{Discussion}

Swimming pace has an impact on performance and there are some studies that have researched the distribution of the split times during pacing (de Koning et al., 2011; Skorski et al., 2013, 2014; Wu et al., 2015). This is the first study that has evaluated the effects of the real time voice feedback modality on swimming pace. Previous research studied audible bleep feedback (Thompson et al., 2002, 2004), but not human voice feedback. Also, the obtained data was compared with other feedback modalities, and this ensured additional relevance to the results. 


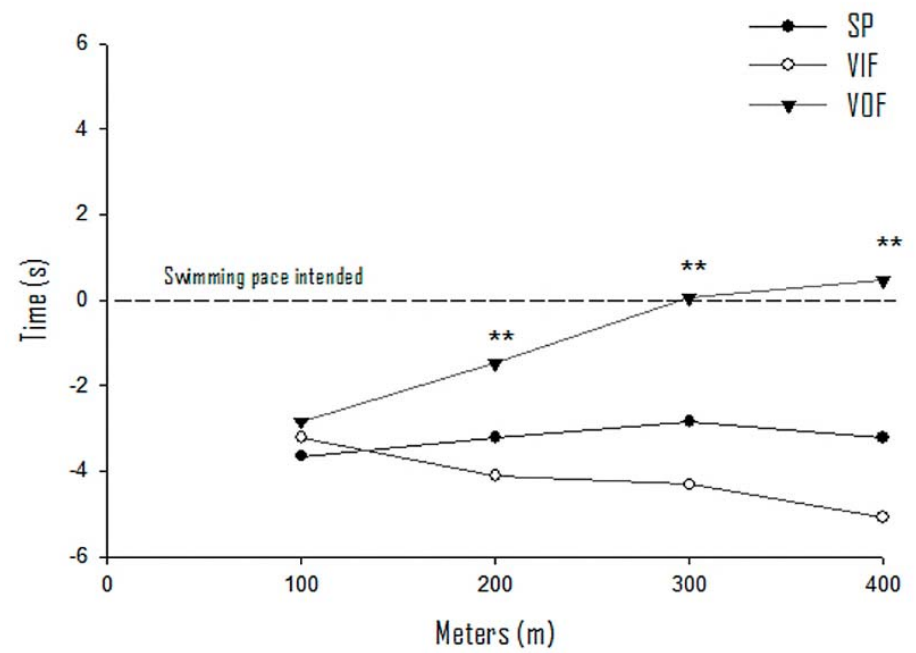

Figure 1

Swimming pace in three different feedback modalities

The graph shows the mean differences in seconds from zero (swimming pace intended) throughout the three $400 \mathrm{~m}$ trials: real time voice feedback (black triangles), real time visual

feedback (white circles) and self-pacing (black circles). Student's t test ${ }^{* *} p<.01$; non-parametric Wilcoxon test ${ }^{* *} p<.01$. SP: Self pacing; VIF: real time visual feedback; VOF: real time voice feedback.

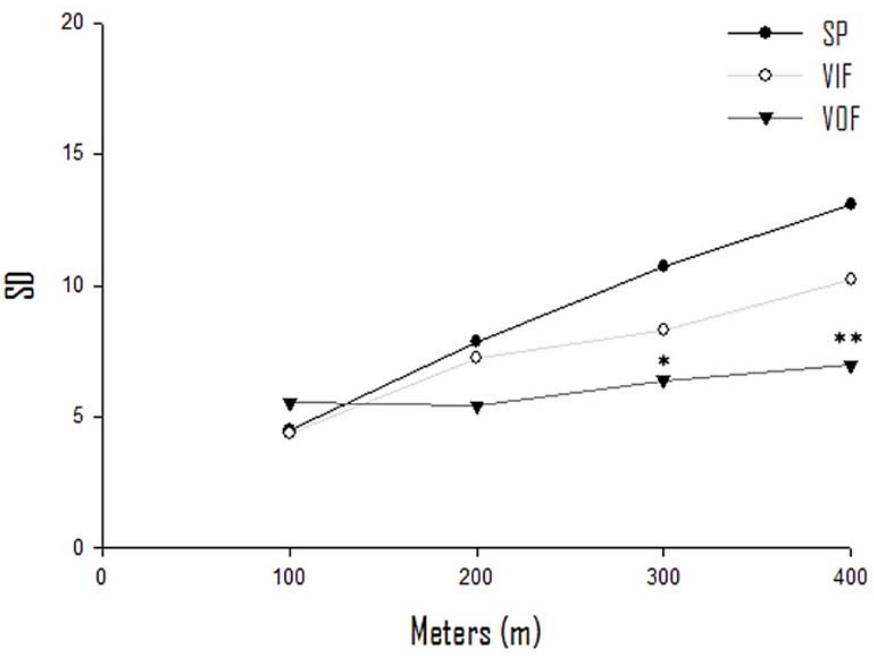

Figure 2

Standard deviation (SD) of swimming pace in three different modalities of feedback The graph shows the mean values of SD throughout the three $400 \mathrm{~m}$ trials: real time voice feedback (black triangles), real time visual feedback (white circles) and self-pacing (black circles). During the trials the SD was more stable in real time voice feedback, as compared to self-pacing and real time visual feedback. The graph shows significant difference between self-pacing and real time voice feedback at $300 \mathrm{~m}$ and $400 \mathrm{~m}$. F-test ${ }^{*} p<.05,{ }^{* *} p<.01$; SP: Self pacing;

VIF: real time visual feedback; VOF: real time voice feedback. 


\section{Table 1}

Overall results of the mean differences in seconds from zero (swimming pace intended) throughout the three $400 \mathrm{~m}$ trials, as well as the absolute differences among feedback modalities

\begin{tabular}{|c|c|c|c|c|c|c|c|c|}
\hline & 100m (SD) & $200 \mathrm{~m}(\mathrm{SD})$ & $300 \mathrm{~m}$ (SD) & $400 \mathrm{~m}$ (SD) & & 100 vs. $200 \mathrm{~m}$ & 100 vs $300 \mathrm{~m}$ & 100 vs. $400 \mathrm{~m}$ \\
\hline SP & $-3.65(1.13)$ & $-3.21(1.97)$ & $-2.84(2.68)$ & $-3.21(3.27)$ & $\begin{array}{c}\text { Dif. } \\
\text { (Sig.) }\end{array}$ & $0.44(0.53)$ & $0.81(0.67)$ & $0.44(0.79)$ \\
\hline$(95 \% \mathrm{CI})$ & $(-6.06,-1.24)$ & $(-7.40,0.98)$ & $(-8.55,2.87)$ & $(-10.18,3.75)$ & ES & 0.12 & 0.12 & 0.05 \\
\hline VIF & $-4.73(1.10)$ & $-4.11(1.81)$ & $-4.32(2.07)$ & $-5.08(2.56)$ & $\begin{array}{l}\text { Dif. } \\
\text { (Sig.) }\end{array}$ & $0.61(0.53)$ & $0.41(0.88)$ & $0.36(0.68)$ \\
\hline$(95 \% \mathrm{CI})$ & $(-7.06,-2.39)$ & $(-7.98,-0.25)$ & $(-8.73,0.10)$ & $(-10.53,0.37)$ & ES & 0.17 & 0.08 & 0.05 \\
\hline VOF & $-2.87(1.39)$ & $-1.47(1.36)$ & 0.05 (1.59) & $0.46(1.74)$ & $\begin{array}{l}\text { Dif. } \\
\text { (Sig.) }\end{array}$ & $1.40(<0.01)$ & $2.92(<0.01)$ & $3.33(0.01)$ \\
\hline$(95 \% \mathrm{CI})$ & $(-5.83,-0.09)$ & $(-4.37,1.41)$ & $(-3.35,3.45)$ & $(-3.25,4.18)$ & ES & 0.79 & 0.81 & 0.76 \\
\hline
\end{tabular}

Absolute Differences among feedback modalities

\begin{tabular}{lcccccccc} 
& 100m (Sig.) & ES & 200m (Sig.) & ES & 300m (Sig.) & ES & 400m (Sig.) & ES \\
\hline SP vs. VIF & $1.08(0.47)$ & 0.17 & $0.90(0.71)$ & 0.13 & $1.48(0.68)$ & 0.17 & $1.87(0.87)$ & 0.18 \\
SP vs. VOF & $0.78(0.65)$ & 0.25 & $1.74(0.41)$ & 0.24 & $2.89(0.39)$ & 0.27 & $3.67(0.66)$ & 0.27 \\
VIF vs. VOF & $1.85(0.44)$ & 0.37 & $2.64(0.19)$ & 0.42 & $4.37(0.09)$ & 0.54 & $5.55(0.12)$ & 0.52 \\
\hline
\end{tabular}

SP: Self pacing; VIF: real time visual feedback; VOF: real time voice feedback.

SD: Standard Deviation. 95\% CI: Interval confidence;

Dif: difference. Sig. Significance. ES: Effect Size.

Table 2

Overall results of standard deviation throughout the three $400 \mathrm{~m}$ trials.

\begin{tabular}{|c|c|c|c|c|c|c|c|c|}
\hline & SD $100 \mathrm{~m}$ & SD $200 \mathrm{~m}$ & $\mathrm{SD} 300 \mathrm{~m}$ & $\mathrm{SD} 400 \mathrm{~m}$ & & $\begin{array}{c}100 \text { vs. } \\
200 \mathrm{~m}\end{array}$ & $\begin{array}{c}200 \text { vs. } \\
300 \mathrm{~m}\end{array}$ & $\begin{array}{c}300 \text { vs. } \\
400 \mathrm{~m}\end{array}$ \\
\hline SP & 4.52 & 7.86 & 10.72 & 13.07 & $\begin{array}{c}\text { F. } \\
\text { (Sig.) }\end{array}$ & $3.02(0.02)$ & $1.86(0.12)$ & $1.49(0.23)$ \\
\hline VIF & 4.39 & 7.25 & 8.28 & 10.23 & $\begin{array}{c}\text { F. } \\
\text { (Sig.) }\end{array}$ & $2.73(0.03)$ & $1.30(0.31)$ & $1.53(0.21)$ \\
\hline VOF & 5.55 & 5.42 & 6.38 & 6.98 & $\begin{array}{c}\text { F. } \\
\text { (Sig.) }\end{array}$ & $0.95(0.54)$ & $1.39(0.27)$ & $1.20(0.37)$ \\
\hline
\end{tabular}

F Snedecor among feedback modalities

\begin{tabular}{ccccc} 
& F. 100m (Sig.) & F. 200m (Sig.) & F. 300m (Sig.) & F. 400m (Sig.) \\
\hline SP vs. VIF & $1.06(0.46)$ & $1.18(0.38)$ & $1.68(0.16)$ & $1.63(0.18)$ \\
SP vs. VOF & $1.51(0.22)$ & $2.10(0.08)$ & $2.82(0.03)$ & $3.51(0.01)$ \\
VIF vs. VOF & $1.60(0.19)$ & $1.79(0.14)$ & $1.68(0.16)$ & $2.15(0.08)$ \\
\hline
\end{tabular}

SD: standard deviation. F: Snedecor F-test. Sig: Significant difference. SP: Self pacing; VIF: real time visual feedback; VOF: real time voice feedback. 
In swimming, the modalities of selfpacing and real time visual feedback are used during training with different distances and different pace. Daily use of these modalities during training could exclude or minimize any learning effect. Our results showed that it was not an easy task for non-elite swimmers to control their own pace by themselves. Previous studies have shown that elite swimmers are able to reproduce accurate swimming pace (Skorski et al., 2014), however, this does not appear to be as precise as a coach might expect (Thompson et al., 2002). Many swimmers are not elite athletes at the top of the Fédération International de Natation (FINA) or International Triathlon Union (ITU) ranking points and our results could have a wide impact on the swimming world including youth athletes. The swimmers adopted a fast start and this finding reinforces the statement that in swimming the "feeling" about your own performance is usually inaccurate; in addition, our results about a fast start are in accordance with previous studies (Mauger et al., 2012; Skorski et al., 2013, 2014). Nonetheless, it is still unclear why the swimmers adopted a fast start.

In any sport performance, control of pacing seems to be a combination of intrinsic (Latash, 1998) and extrinsic feedback (Swinnen, 1990). In swimming, extrinsic feedback factors could play a crucial role in regulating eventual changes if there are self-misperceptions. A previous study has shown that different feedback conditions could affect the control of swimming speed (Perez et al., 2009). Real time feedback is an input of great value for training, it allows immediate feedback and this is more effective than delayed feedback (Grosser and Neumaier, 1986).

Previous studies have demonstrated that different types of feedback can improve swimming pace control and other swimming variables (Bächlin and Tröster, 2012; Hagem et al., 2013a, b; Perez et al., 2009). The audio feedback modality has been shown to be useful in swimming (Thompson et al., 2002; Turner et al., 2008). Our results revealed that the real time voice feedback modality, via the Ucoach device, greatly helped the swimmers in controlling the swimming pace. Data showed that the swimmers maintained consistent pace when the real time voice feedback was given. We know that the auditory system is preferential in the marine mammal group (Ketten, 2002); based on our results and previous studies on audio feedback (Thompson et al., 2002, 2004), the auditory system could be a preferred way to communicate with swimmers in the aquatic environment. The obtained data might be considered important for the development of a training workout in the aerobic range where the real time voice feedback could be very useful. Further research should be conducted with faster swimming pace close to competition pace. Some recorded differences may not only have statistical significance, but they could be especially interesting for coaches. Our sample was heterogeneous, thus further research with real time voice feedback is necessary in elite and inexperienced swimmers. Moreover, future studies should be performed also with the other three swimming styles (backstroke, breaststroke and butterfly).

SD values were stable during the trials and showed that the real time voice feedback, given via the Ucoach device, was the most accurate feedback modality among the three ones used in this study. We strongly believe that Ucoach is a useful device for swimming pace training, as well as any other tool that allows speaking with swimmers during in-water training.

In this study, communication with swimmers was regarding their swimming pace, without giving any other additional information. However, it is thought that using the Ucoach device, the coach could give many other short messages to swimmers regarding other aspects of performance, such as technique, body posture, body rotation in crawl and backstroke, stroke efficiency, leg kicks, feedback during turns and rhythm or breathing cycle changes and any other aspects considered important for coaches and swimmers. Real time voice feedback via the Ucoach device could also be useful when swimmers swim in open water, which is a more difficult training environment. The real time voice feedback could be used in other aquatic sports such as synchronized swimming and lifesaving sport.

This study demonstrates that real time voice feedback could be an accurate modality for helping swimmers in maintaining their swimming pace. In this regard, the swimmers had a good 
response after real time voice feedback and they reached stable pacing as desired. Compared with other modalities, the real time voice feedback could be the best way to manage swimming pace. The real time voice feedback seems to be an effective way to communicate changes in swimming pace during training. Swimmers became immediately familiar with the Ucoach device and it appeared that the communication provided via the auditory system allowed an accurate and reliable modality for controlling swimming pace. The study shows that real time voice feedback by the Ucoach device made the communication with the swimmers effective and easy, and this tool has wide applications in swimming, not only in swimming pace. Moreover, with the real time voice feedback the coach could motivate and encourage the swimmers for a greater commitment and provide them with continuous technical corrections from the poolside.

\section{Acknowledgements}

The Ucoach device was kindly given by SHOT (Swimmers Hit On Technology, Rome, Italy). This research did not receive any specific grant from funding agencies in the public, commercial, or non-profit sectors.

\section{References}

Abbiss CR, Ross ML, Garvican LA, Ross N, Pottgiesser T, Gregory J, Martin DT. The distribution of pace adopted by cyclists during a cross-country mountain bike World Championships. J Sports Sci, 2013; 31(7): 787-794. https://doi.org/10.1080/02640414.2012.751118

Bächlin M, Tröster G. Swimming performance and technique evaluation with wearable acceleration sensors. Pervasive Mob Comput, 2012; 8(1): 68-81. http://dx.doi.org/10.1016/j.pmcj.2011.05.003

Bath D, Turner LA, Bosch AN, Tucker R, Lambert EV, Thompson KG, St Clair Gibson A. The effect of a second runner on pacing strategy and RPE during a running time trial. Int J Sports Physiol Perform, 2012; 7(1): 26-32. https://doi.org/2011-0045

Cohen J. A power primer. Psychol Bull, 1992; 112(1): 155-9. http://www.ncbi.nlm.nih.gov/pubmed/19565683

de Koning JJ, Foster C, Bakkum A, Kloppenburg S, Thiel C, Joseph T, Porcari JP. Regulation of pacing strategy during athletic competition. PloS One, 2011; 6(1): e15863. https://doi.org/10.1371/journal.pone.0015863

Dekerle J, Sidney M, Hespel JM, Pelayo P. Validity and reliability of critical speed, critical stroke rate, and anaerobic capacity in relation to front crawl swimming performances. Int J Sports Med, 2002; 23(2); 93 98. https://doi.org/10.1055/s-2002-20125

Edwards AM, Polman RC. Pacing in Sport and Exercise. Nova Science Publisher New York., Inc., 1-208; 2012

Ginn E. Critical speed and training intensities for swimming. A report presented to the Australian Sports Commission's applied Sport Research Program; 1993

Grosser M, Neumaier A. Técnicas de entrenamiento: teoría y práctica de los deportes. B. Martínez Roca, Ed., 1-168; 1986

Hagem RM, Haelsig T, O'Keefe SG, Stamm A, Fickenscher T, Thiel DV. Second Generation Swimming Feedback Device Using a Wearable Data Processing System based on Underwater Visible Light Communication. 6th Asia-Pacific Congress on Sports Technology (APCST), 2013; 60: 34-39. http://dx.doi.org/10.1016/j.proeng.2013.07.065

Hagem RM, O'Keefe SG, Fickenscher TTDV. Self-contained adaptable optical wireless communications system for stroke rate during swimming. IEEE Sens J, 2013; 13: 3144-3151

Ketten DR. Marine Mammal Auditory Systems: A Summary ofAudiometric and Anatomical Data and Implications for Underwater Acoustic Impacts. Polarforschung, 2002; 72 (2/3): 79-92

Latash M. Neurophysiological Basis of Movement-2nd edition (Human Kinetics) Champaign, 1-440; 1998 
Le Meur Y, Hausswirth C, Dorel S, Bignet F, Brisswalter J, Bernard T. Influence of gender on pacing adopted by elite triathletes during a competition. Eur J App Physiol, 2009; 106(4): 535-545. https://doi.org/10.1007/s00421-009-1043-4

Mauger AR, Neuloh J, Castle PC. Analysis of pacing strategy selection in elite 400-m freestyle swimming. Med Sci Sport Exer, 2012; 44(11): 2205-2212. https://doi.org/10.1249/MSS.0b013e3182604b84

Mytton GJ, Archer DT, Turner L, Skorski S, Renfree A, Thompson KG, St Clair Gibson A. Increased variability of lap speeds: differentiating medalists and nonmedalists in middle-distance running and swimming events. Int J Sports Physiol Perform, 2015; 10(3): 369-73. https://doi.org/10.1123/ijspp.20140207

Perez P, Llana S, Brizuela G, Encarnacion A. Effects of three feedback conditions on aerobic swim speeds. J Sport Sci Med, 2009; 8(1): 30-36

Scruton A, Baker J, Roberts J, Basevitch I, Merzbach V, Gordon D. Pacing accuracy during an incremental step test in adolescent swimmers. Open Acc J Sport Med, 2015; 6: $249-57$. https://doi.org/10.2147/OAJSM.S84906

Skorski S, Faude O, Caviezel S, Meyer T. Reproducibility of pacing profiles in elite swimmers. Int J Sports Physiol Perform, 2014; 9(2): 217-225. https://doi.org/10.1123/ijspp.2012-0258

Skorski S, Faude O, Rausch K, Meyer T. Reproducibility of pacing profiles in competitive swimmers. Int J Sports Med, 2013; 34(2): 152-157. https://doi.org/10.1055/s-0032-1316357

Swinnen SP. Interpolated activities during the knowledge-of-results delay and post-knowledge-of-results interval: Effects on performance and learning. J Exp Psychol Learn, 1990; 16(4): 692-705

Thompson KG. Pacing - Individual Strategies for Optimal Performance. Human Kinetics, 1-240; 2014

Thompson KG, MacLaren DP, Lees A, Atkinson G. Accuracy of pacing during breaststroke swimming using a novel pacing device, the Aquapacer. J Sports Sci, 2002; 20(7): 537-546. https://doi.org/10.1080/026404102760000044

Thompson KG, MacLaren DP, Lees A, Atkinson G. The effects of changing pace on metabolism and stroke characteristics during high-speed breaststroke swimming. J Sports Sci, 2004; 22(2): 149-157. https://doi.org/10.1080/02640410310001641467

Touretski G. Footprints to Success. ASCA Newsletter, 1993; 10(6): 5-14

Tucker R, Noakes TD. The physiological regulation of pacing strategy during exercise: a critical review. Brit J Sport Med, 2009; 43(6): e1. https://doi.org/10.1136/bjsm.2009.057562

Turner AP, Smith T, Coleman SG. Use of an audio-paced incremental swimming test in young national-level swimmers. Int J Sports Physiol Perform, 2008; 3(1): 68-79

Wu SS, Peiffer JJ, Brisswalter J, Nosaka K, Lau WY, Abbiss CR. Pacing strategies during the swim, cycle and run disciplines of sprint, Olympic and half-Ironman triathlons. Eur J App Physiol, 2015; 115(5): 11471154. https://doi.org/10.1007/s00421-014-3096-2

\section{Corresponding author:}

\section{Roberto Cejuela}

Department-section of Physical Education and Sport, University of Alicante, Alicante, Spain, Campus de San Vicente del Raspeig. 03540. San Vicente del Raspeig (Alicante), Spain

Tel: +34.965903400

Fax : +34.965903464

Email : roberto.cejuela@ua.es 